\title{
Four Parameters Kumaraswamy Reciprocal Family Of Distributions
}

\author{
Salma Omar Bleed ${ }^{1^{*}}$ \\ ${ }^{1}$ College of Science, Statistics Department, Al-asmarya Islamic University, Zliten- Libya
}

\begin{abstract}
In this paper, kumaraswamy reciprocal family of distributions is introduced as a new continues model with some of approximation to other probabilistic models as reciprocal, beta, uniform, power function, exponential, negative exponential, weibull, rayleigh and pareto distribution. Some fundamental distributional properties, force of mortality, mills ratio, bowley skewness, moors kurtosis, reversed hazard function, integrated hazard function, mean residual life, probability weighted moments, bonferroni and lorenz curves, laplace-stieltjes transform of this new distribution with the maximum likelihood method of the parameter estimation are studied. Finally, four real data sets originally presented are used to illustrate the proposed estimators.
\end{abstract}

Keywords: Kumaraswamy distribution, Reciprocal distribution, Force of mortality, Mills Ratio, Bowley skewness, Moors kurtosis, Reversed hazard function, Integrated hazard function, Mean residual life, Probability weighted moments, Bonferroni and Lorenz curves, Laplace-Stieltjes transform.

*S.Ali@asmary.edu.ly \& Salmableed@yahoo.com 


\section{Introduction}

The reciprocal distribution is a continuous probability distribution, gets its name because the density function is proportional to $x^{-I}$. It is a right-skewed distribution bounded between min (a) and max (b), and it is looks like a triangle (min, mode, max), if the min and the max are almost equal. Also, it is an example of an inverse distribution where the reciprocal of a random variable with a reciprocal distribution itself has a reciprocal distribution.

The probability density function (pdf) of the reciprocal distribution is

$$
f(x)=A x^{-1}, a \leq x \leq b, b>a>0
$$

with cumulative distribution function $F(x)=A Q(x, a) \quad a \leq x \leq b, b>a>0$

$$
\text { where, } A=(\ln b-\ln a)^{-1}, Q(x, a)=(\ln x-\ln a) \text {. }
$$

The reciprocal distribution is of considerable importance in numerical analysis as a computer's arithmetic operations transform mantissas with initial arbitrary distributions to the reciprocal distribution as a limiting distribution. Also, it is widely used as an uninformed prior distribution in Bayesian inference for scale parameters, Hamming (1970) .

In the last few years, many new generated families are formed by adding additional one or more shape parameters to develop new models and increase its flexibility, such as Quasi Lindley distribution (Rama and Mishra, 2013), Kumaraswamy Weibull-generated family of distributions (Hassan, and Elgarhy, 2016), Kumaraswamy sushila distribution (Shawki and Elgarhy, 2017), L-Quadratic distribution (Salma, 2018), TAS distribution (Salma and Arwa, 2018), and others. The Kumaraswamy distribution is one of them, it has the following density function with two additional shape parameters $\alpha$ and $\beta$

with distribution function $G(x)=\left[1-F(x)^{\alpha}\right]^{\beta}$

$$
g(x)=\alpha \beta f(x) F(x)^{\alpha-1}\left[1-F(x)^{\alpha}\right]^{\beta-1}, \quad \alpha, \beta>0
$$

It is well known in general that, a generalized model is having more flexibility than the base model and it is favored by data analysts in analyzing data. Therefore, the main objective of this paper to propose a new continuous kumaraswamy reciprocal family and explain its flexible behavior, and how it is fitted for various continuous data sets in different applied fields. Section (2) identifies the four parameters kumaraswamy reciprocal family with some of its approximation to other probabilistic models and studding its reliability measurements behavior. Section (3) contains the properties of the kumaraswamy reciprocal family and the Maximum Likelihood (ML) method of the parameter estimation. Section (4) presents the practical applications to illustrate the proposed model.

\section{The Four Parameters Kumaraswamy Reciprocal Family}

The new distribution will abbreviate by $\operatorname{KR}(a, b, \alpha, \beta)$ with probability function takes the form

$$
f(x)=\alpha \beta x^{-1} A^{\alpha} Q(x, a)^{\alpha-1}\left[1-A^{\alpha} Q(x, a)^{\alpha}\right]^{\beta-1}, a \leq x \leq b, b>a>0 \rightarrow(1)
$$

with cumulative distribution function $F(x)=1-\left[1-A^{\alpha} Q(x, a)^{\alpha}\right]^{\beta}, \rightarrow(2)$ where, $A=(\ln b-\ln a)^{-1}$,

$$
Q(x, a)=(\ln x-\ln a) .
$$



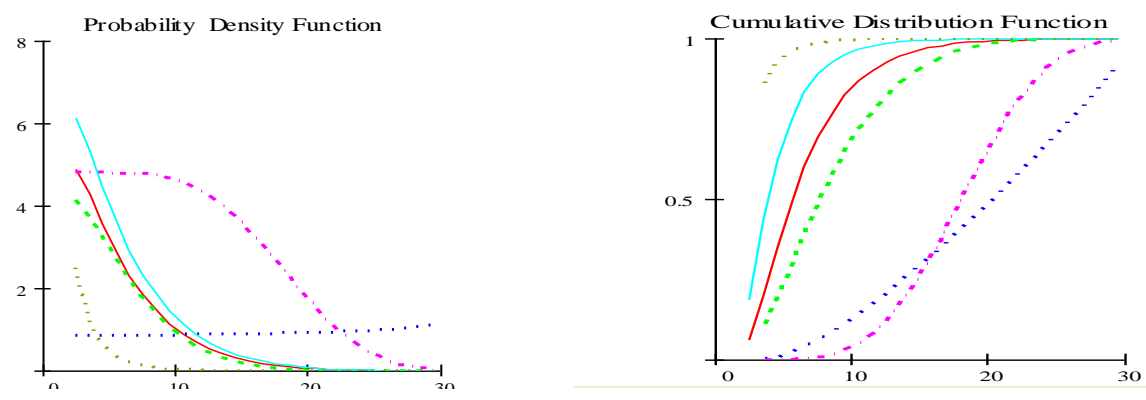

Figure 1: PDF and $C D F$ of $\operatorname{KRF}(a, b, \alpha, \beta)$

Figure. 1 illustrate plot the PDF and the CDF of the $K R F(a, b, \alpha, \beta)$ under different values of the shape parameters $(\alpha, \beta)$ and the scale parameters $(a, b)$. It is noted that the PDF of the new distribution takes many shapes, and the plot of the CDF indicates increasing cumulative distribution function.

\subsection{Approximation to other probabilistic models}

Some of approximation to other probabilistic models are presented in Table.1 as some special cases of $\operatorname{KRF}(a, b, \alpha, \beta)$.

\begin{tabular}{|c|c|c|c|}
\hline Parameters & Random Variable & Distributed as & Density Functions \\
\hline$\alpha=\beta=1$ & $X$ & Reciprocal & $f(x)=A x^{-1}, a \leq x \leq b, b>a>0$ \\
\hline$\beta$ & $y=A^{\alpha} Q(x, a)^{\alpha}$ & $\operatorname{Beta}(1, \beta)$ & $f(y)=\frac{1}{(1, \beta)}(1-y)^{\beta-1}, \quad 0<y<1, \quad \beta>0$ \\
\hline$\beta=1$ & $y=A^{\alpha} Q(x, a)^{\alpha}$ & Uniform & $f(y)=1 \quad, \quad 0<y<1$ \\
\hline$\beta$ & $y=1-A^{\alpha} Q(x, a)^{\alpha}$ & Power Function & $f(y)=\beta y^{\beta-1}, \quad 0<y<1, \quad \beta>0$ \\
\hline$\beta=\frac{1}{\theta}$ & $y=-\ln \left\{1-A^{\alpha} Q(x, a)^{\alpha}\right\}$ & Exponential & $f(y)=\frac{1}{\beta} e^{\frac{-1}{\beta} y}, y>0, \theta>0$ \\
\hline$\beta$ & $y=-\ln \left\{1-A^{\alpha} Q(x, a)^{\alpha}\right\}$ & -ve Exponential & $f(y)=\beta e^{-\beta y}, y>0, \beta>0$ \\
\hline$\beta, K$ & $y=k\left\{1-A^{\alpha} Q(x, a)^{\alpha}\right\}^{-1}$ & Pareto & $f(y)=\frac{\beta k^{\beta}}{y^{\beta+1}}, y \geq k, \quad \beta, k>O$ \\
\hline$\alpha=\frac{1}{\lambda}$ & $y^{\frac{1}{\alpha}}=-\ln \left\{1-A^{\alpha} Q(x, a)^{\alpha}\right\}$ & Weibull & $f(y)=\frac{\beta}{\alpha} y^{\frac{1}{\alpha}-1} e^{\frac{-\beta}{\alpha} y^{\frac{1}{\alpha}}}, y>O, \lambda . \beta>O$ \\
\hline$\alpha=\frac{1}{2}, \beta=\frac{1}{2 \alpha^{2}}$ & $y^{\frac{1}{\alpha}}=-\ln \left\{1-A^{\alpha} Q(x, a)^{\alpha}\right\}$ & Rayleigh & $f(y)=\frac{1}{\alpha^{2}} y e^{\frac{-1}{\alpha^{2} y}}, y>O, \beta>O$ \\
\hline
\end{tabular}

\subsection{Maximum likelihood estimation}

The reliability function (RF) of the four parameters $K R F(a, b, \alpha, \beta)$ is

$$
R(x)=1-F(x)=\left[1-A^{\alpha} Q(x, a)^{\alpha}\right]^{\beta}
$$

The force of mortality (FM) of the four parameters $\operatorname{KRF}(a, b, \alpha, \beta)$ is

$$
h(x)=\frac{f(x)}{R(x)}=\frac{\alpha \beta}{x Q(x, a)\left[A^{-\alpha} Q(x, a)^{-\alpha}-1\right]}
$$

The reversed hazard function (RHF) of the four parameters $\operatorname{KRF}(a, b, \alpha, \beta)$ is 


$$
R_{h}(x)=\frac{f(x)}{F(x)}=\frac{\alpha \beta\left[A^{-\alpha} Q(x, a)^{-\alpha}-1\right]^{-1}}{x Q(x, a)^{\alpha-1}\left[\left[1-A^{\alpha} Q(x, a)^{\alpha}\right]^{-\beta}-1\right]}
$$

The integrated hazard function (IHF) of the four parameters $K R F(a, b, \alpha, \beta)$ is

$$
H(x)=-\ln R(x)=-\beta \ln \left[1-A^{\alpha} Q(x, a)^{\alpha}\right]
$$

The mills ratio (MR) of the four parameters $\operatorname{KRF}(a, b, \alpha, \beta)$ is

$$
\operatorname{mi}(x)=\frac{1}{h(x)}=\frac{x\left\lfloor A^{-\alpha} Q(x, a)^{-\alpha}-1\right\rfloor}{\alpha \beta Q(x, a)} .
$$

\section{Properties of the Kumaraswamy Reciprocal Family}

\subsection{Quantile Function}

The quantile function $Q(u)$ of the four parameters $\operatorname{KRF}(a, b, \alpha, \beta)$ has closed form and is obtained by inverting Eq.(2), therefore

$$
Q(u)=a \exp \left\{A^{-1}\left[1-(1-u)^{\frac{1}{\beta}}\right]^{\frac{1}{\alpha}}\right\} \rightarrow(3)
$$

\subsection{Median and Random Numbers}

From Eq.(3), if u equal to 0.5 then the median of $\operatorname{KRF}(a, b, \alpha, \beta)$ is

$$
m=a \exp \left\{A^{-1}\left[1-0.5^{\beta^{-1}}\right]^{\frac{1}{\alpha}}\right\} .
$$

Also, from Eq.(3), if $u \sim U(0,1)$ and the parameters ${ }^{(a, b, \alpha, \beta)}$ are known, then the random numbers of $\operatorname{KRF}(a, b, \alpha, \beta)$ can be generated.

\subsection{Bowley Skewness and Moors Kurtosis}

The Bowley Skewness (BS), and Moors Kurtosis (MK) of the four parameters $\operatorname{KRF}(a, b, \alpha, \beta)$ are obtained as follows

$B S=\frac{Q(0.75)-2 Q(0.5)+Q(0.25)}{Q(0.75)-Q(0.25)}, \quad M K=\frac{Q(0.875)-Q(0.625)+Q(0.375)-Q(0.125)}{Q(0.75)-Q(0.25)}$

Where $Q(u)$ is the quantile function of the four parameters $\operatorname{KRF}(a, b, \alpha, \beta)$.

\subsection{Mean Residual Life}

Theorem 1: The mean residual life of a r.v $X$ with pdf (1), is given by $\mu(t)=\frac{a}{\alpha R(t)} \sum_{\zeta=1}^{\infty} \frac{1}{A^{\zeta+\alpha} \zeta !} I_{I-T}\left(\beta+1, \frac{\zeta+1}{\alpha}\right), T=A^{\alpha}(\ln t-\ln a)^{\alpha}$

Proof: The Mean residual life of a $\operatorname{r.v} X$ is defined as $\mu(t)=\frac{1}{R(t)} \int_{t}^{b} R(x) d x$, then 


$$
\begin{aligned}
& \frac{1}{R(t)} \int_{t}^{b} R(x) d x=\frac{1}{R(t)} \int_{t}^{b}\left[1-A^{\alpha} Q(x, a)^{\alpha}\right]^{\beta} d x \\
& =\frac{a}{\alpha A R(t)} \int_{T}^{1} y^{\frac{1}{\alpha}-1}[1-y]^{\beta} e^{\frac{y^{\alpha}}{A}} d y, \text { such that } y=A^{\alpha} Q(x, a)^{\alpha}, \quad T=A^{\alpha}(\ln t-\ln a)^{\alpha} .
\end{aligned}
$$

For $n, m>0$ integer, it follows that

$$
\int_{a}^{1} w^{n-1}[1-w]^{m-1} e^{w} d w=\sum_{\zeta=1}^{\infty} \frac{I_{1-a}(n+\zeta, m)}{\zeta !} \rightarrow(4)
$$

where $I_{1-a}(a, b)=\int_{a}^{l} w^{a-1}[1-w]^{b-1} d w$ denotes the complementary incomplete beta function which can be evaluated in MathCad 15. Using Eq.(4), the mean residual life can be expressed as

$$
\mu(t)=\frac{a}{\alpha R(t)} \sum_{\zeta=1}^{\infty} \frac{1}{A^{\zeta+\alpha} \zeta !} I_{1-T}\left(\beta+1, \frac{\zeta+1}{\alpha}\right), T=A^{\alpha}(\ln t-\ln a)^{\alpha} .
$$

\subsection{Moments about Origin}

Theorem 2: If $\mathrm{X}$ is a r.v distributed as $\operatorname{KRF}(a, b, \alpha, \beta)$, then $\mathrm{r}^{\text {th }}$ moments of $\mathrm{X}$ is given by

$$
\mu_{r}^{\prime}=a^{r}+\frac{a^{r}}{\alpha} \sum_{\zeta=1}^{\infty} \frac{r^{\zeta+1}}{A^{\zeta+1} \zeta !} \beta\left(\frac{\zeta+1}{\alpha}, \beta+1\right)
$$

Proof: $\mathrm{r}^{\text {th }}$ moments is defined as $\mu_{r}^{\prime}=\int_{a}^{b} x^{r} f(x) d x$, then

$$
\int_{a}^{b} x^{r} f(x) d x=\beta \int_{a}^{b} \frac{\alpha x^{r}}{x} A^{\alpha} Q(x, a)^{\alpha-1}\left[1-A^{\alpha} Q(x, a)^{\alpha}\right]^{\beta-1} d x .
$$

By using the partial derivative

$$
\int_{a}^{b} x^{r} f(x) d x=(-\beta)\left[\left.\frac{x^{r}\left[1-A^{\alpha} Q(x, a)^{\alpha}\right]^{\beta}}{\beta}\right|_{a} ^{b}-\frac{r}{\beta} \int_{a}^{b} x^{r-1}\left[1-A^{\alpha} Q(x, a)^{\alpha}\right]^{\beta} d x\right]=a^{r}+\frac{r a^{r}}{\alpha A} \int_{0}^{1} y^{\frac{1}{\alpha}-1}[1-y]^{\beta} e^{\frac{r y \alpha}{A}} d y \cdot y=A^{\alpha} Q(x, a)^{\alpha}
$$

For $n, m>0$ integer, it follows that

$$
\int_{0}^{1} w^{n-1}[1-w]^{m-1} e^{w} d w=\sum_{\zeta=1}^{\infty} \frac{\beta(n+\zeta, m)}{\zeta !} \rightarrow(5)
$$

where

$$
\beta(a, b)=\int_{0}^{1} w^{a-1}[1-w]^{b-1} d w=\frac{\Gamma a \Gamma b}{\Gamma(a+b)} \text { denotes the beta function. }
$$

Using Eq.(5), the $\mathrm{r}^{\text {th }}$ moments can be expressed as

$$
\mu_{r}^{\prime}=a^{r}+\frac{a^{r}}{\alpha} \sum_{\zeta=1}^{\infty} \frac{r^{\zeta+1}}{A^{\zeta+1} \zeta !} \beta\left(\frac{\zeta+1}{\alpha}, \beta+1\right), r=1,2, \ldots \rightarrow(6) .
$$

From Eq.(6), the $1^{\text {st }}$ four moments and the variance of the $\operatorname{KRF(a,b,\alpha ,\beta )}$ are

$$
\mu=\mu_{1}^{\prime}=a+\frac{a}{\alpha} \sum_{\zeta=1}^{\infty} \frac{\beta\left(\frac{\zeta+1}{\alpha}, \beta+1\right)}{A^{\zeta+1} \zeta !}, \mu_{2}^{\prime}=a^{2}+\frac{a^{2}}{\alpha} \sum_{\zeta=1}^{\infty} \frac{2^{\zeta+1}}{A^{\zeta+1} \zeta !} \beta\left(\frac{\zeta+1}{\alpha}, \beta+1\right)
$$




$$
\begin{gathered}
\mu_{3}^{\prime}=a^{3}+\frac{a^{3}}{\alpha} \sum_{\zeta=1}^{\infty} \frac{3^{\zeta+1}}{A^{\zeta+1} \zeta !} \beta\left(\frac{\zeta+1}{\alpha}, \beta+1\right), \mu_{4}^{\prime}=a^{4}+\frac{a^{4}}{\alpha} \sum_{\zeta=1}^{\infty} \frac{4^{\zeta+1}}{A^{\zeta+1} \zeta !} \beta\left(\frac{\zeta+1}{\alpha}, \beta+1\right) \\
\sigma^{2}=\left[a^{2}+\frac{a^{2}}{\alpha} \sum_{\zeta=1}^{\infty} \frac{2^{\zeta+1}}{A^{\zeta+1} \zeta !} \beta\left(\frac{\zeta+1}{\alpha}, \beta+1\right)\right]-\left[a+\frac{a}{\alpha} \sum_{\zeta=1}^{\infty} \frac{\beta\left(\frac{\zeta+1}{\alpha}, \beta+1\right)}{A^{\zeta+1} \zeta !}\right]^{2}
\end{gathered}
$$

Put $\alpha=\beta=1$ then $\mu=A(b-a)$ and $\sigma^{2}=\frac{A(b-a)}{2}[(b+a)-2 A(b-a)]$ which are the mean and the variance of the base reciprocal distribution, respectively.

\subsection{Incomplete Moments}

Theorem 3: If $\mathrm{X}$ is a r.v distributed as $\operatorname{KR}(a, b, \alpha, \beta)$, then $\mathrm{r}^{\text {th }}$ incomplete moments of $\mathrm{X}$ is given by

$$
I\left(\mu_{r}^{\prime}\right)=\psi(x ; a, b)+\frac{a^{r}}{\alpha} \sum_{\zeta=1}^{\infty} \frac{r^{\zeta+1}}{A^{\zeta+1} \zeta !} I_{y}\left(\frac{\zeta+1}{\alpha}, \beta+1\right)
$$

Where

$$
\psi(x ; a, b)=a^{r}-x^{r}\left[1-A^{\alpha} Q(x, a)^{\alpha}\right]^{\beta} \text {, and } y=A^{\alpha} Q(x, a)^{\alpha} .
$$

Proof: $\mathrm{r}^{\text {th }}$ incomplete moments is defined as $I\left(\mu_{r}^{\prime}\right)=\int_{a}^{x} x^{r} f(x) d x$, then

$$
I\left(\mu_{r}^{\prime}\right)=\int_{a}^{x} x^{r} f(x) d x=\beta \int_{a}^{x} \frac{\alpha x^{r}}{x} A^{\alpha} Q(x, a)^{\alpha-1}\left[1-A^{\alpha} Q(x, a)^{\alpha}\right]^{\beta-1} d x
$$

By using the partial derivative

$$
\int_{a}^{x} x^{r} f(x) d x=(-\beta)\left[\left.\frac{x^{r}\left[1-A^{\alpha} Q(x, a)^{\alpha}\right]^{\beta}}{\beta}\right|_{a} ^{x}-\frac{r}{\beta} \int_{a}^{x} x^{r-1}\left[1-A^{\alpha} Q(x, a)^{\alpha}\right]^{\beta} d x\right]=\psi+\frac{r a^{r} \nabla}{\alpha A} \int_{0}^{\frac{1}{\alpha}-1}[1-y]^{\beta} e^{\frac{r y \frac{1}{\alpha}}{A}} d y, y=A^{\alpha} Q(x, a)^{\alpha}
$$

For $n, m>0$ integer, it follows that

$$
\int_{0}^{b} w^{n-1}[1-w]^{m-1} e^{w} d w=\sum_{\zeta=1}^{\infty} \frac{I_{b}(n+\zeta, m)}{\zeta !} \rightarrow(7)
$$

Where $I_{b}(a, b)=\int_{0}^{\hbar} w^{a-1}[1-w]^{b-1} d w$ denotes the complementary incomplete beta function, which can be evaluated in MathCad 15. Using Eq.(7), the incomplete moments can be expressed as

$$
I\left(\mu_{r}^{\prime}\right)=\psi(x ; a, b)+\frac{a^{r}}{\alpha} \sum_{\zeta=1}^{\infty} \frac{r^{\zeta+1}}{A^{\zeta+1} \zeta !} I_{y}\left(\frac{\zeta+1}{\alpha}, \beta+1\right) .
$$

\subsection{Probability Weighted Moments}

Theorem 4: The probability weighted moments $\left({ }_{r, s}^{\prime}\right)$ of a r.v X distributed as $\operatorname{KRF}(a, b, \alpha, \beta)$ is

$$
\mu_{r, s}^{\prime}=\frac{1}{s+1}\left[b^{r}-\frac{a^{r}}{\alpha} \sum_{i, j=0}^{\infty} \frac{(-1)^{j} r^{i+1}}{A^{i+1} i ! j !}\left(\begin{array}{c}
s+1 \\
j
\end{array}\right) \beta\left(\frac{i+1}{\alpha}, j \beta+1\right)\right]
$$

Proof: The probability weighted moments of a r.v X is defined a $\mu_{r, s}^{\prime}=\int_{a}^{b} x^{r} f(x)[F(x)]^{s} d x$ 
Therefore,

$$
\int_{a}^{b} x^{r} f(x)[F(x)]^{s} d x=\beta \int_{a}^{b} \frac{\alpha x^{r}}{x} A^{\alpha} Q(x, a)^{\alpha-1}\left[1-A^{\alpha} Q(x, a)^{\alpha}\right]^{\beta-1}\left[1-\left[1-A^{\alpha} Q(x, a)^{\alpha}\right]^{\beta}\right]^{s} d x
$$

By using the partial derivative

$$
\int_{a}^{b} x^{r} f(x)[F(x)]^{s} d x=\frac{1}{s+1}\left[b^{r}-r \int_{a}^{b} x^{r-1}\left\{1-\left[1-A^{\alpha} Q(x, a)^{\alpha}\right]^{\beta}\right\}^{s+1} d x\right]
$$

Put $y=A^{\alpha} Q(x, a)^{\alpha}$ and using Eq.(5)

$$
\underset{a}{r} \int^{r} x^{r-1}\left\{1-\left[1-A^{\alpha} Q(x, a)^{\alpha}\right]^{\beta}\right\}^{s+1} d x=\frac{a^{r}}{\alpha} \sum_{i, j=0}^{\infty} \frac{(-1)^{j} r^{i+1}}{A^{i+1} i !}\left(\begin{array}{c}
s+1 \\
j
\end{array}\right) \beta\left(\frac{i+1}{\alpha}, j \beta+1\right)
$$

Therefore,

$$
\mu_{r, s}^{\prime}=\frac{1}{s+1}\left[b^{r}-\frac{a^{r}}{\alpha} \sum_{i, j=0}^{\infty} \frac{(-1)^{j} r^{i+1}}{A^{i+1} i !}\left(\begin{array}{c}
s+1 \\
j
\end{array}\right) \beta\left(\frac{i+1}{\alpha}, j \beta+1\right)\right] .
$$

\subsection{Bonferroni and Lorenz Curves}

Suppose that $Y$ is a count response variable that follows the EWGD in Equation (2.2) and $Y$ is associated with a set of predictors. We wish to fit the response variable $Y$ by using the predictors. Suppose we have a $k-1$ row vector of predictors $x_{i}=\left(x_{i 0}=1, x_{i 1}, x_{i 2}, \ldots, x_{i,(k-1)}\right)$.

Theorem 5: If $\mathrm{X}$ is a r.v distributed as $\operatorname{KRF}(a, b, \alpha, \beta)$, then the Bonferroni and Lorenz curves (BLC) of $\mathrm{X}$ is

$$
B_{F}[F(x)]=\frac{1}{\mu F(x)}\left\{a-x\left[1-A^{\alpha} Q(x, a)^{\alpha}\right]^{\beta}+\frac{a}{\alpha} \sum_{\zeta=1}^{\infty} \frac{I_{y}\left(\frac{\zeta+1}{\alpha}, \beta+1\right)}{A^{\zeta+1} \zeta !}\right\}
$$

Proof: The Bonferroni and Lorenz curves ${ }^{B} F[F(x)]$ is defined by the following formula: $B_{F}[F(x)]=\frac{1}{\mu F(x)} \int_{a}^{x} w f(w) d w$

$$
\begin{aligned}
& \text {. Therefore, } \\
& \frac{1}{\mu F(x)} \int_{a}^{x} w f(w) d w=\beta \int_{a}^{x} \frac{\alpha w}{w} A^{\alpha} Q(w, a)^{\alpha-1}\left[1-A^{\alpha} Q(w, a)^{\alpha}\right]^{\beta-1} d w
\end{aligned}
$$

By using the partial derivative

$$
\frac{1}{\mu F(x)} \int_{a}^{x} w f(w) d w=a-x\left[1-A^{\alpha} Q(x, a)^{\alpha}\right]^{\beta}+\int_{a}^{x}\left[1-A^{\alpha} Q(w, a)^{\alpha}\right]^{\beta} d w
$$

Put $y=A^{\alpha} Q(x, a)^{\alpha}$, and using Eq.(7)

Therefore

$$
\begin{array}{r}
\int_{a}^{x}\left[1-A^{\alpha} Q(w, a)^{\alpha}\right]^{\beta} d w=\frac{a}{\alpha} \sum_{\zeta=1}^{\infty} \frac{I_{y}\left(\frac{\zeta+1}{\alpha}, \beta+1\right)}{A^{\zeta+1} \zeta !} \\
B_{F}[F(x)]=\frac{1}{\mu F(x)}\left\{a-x\left[1-A^{\alpha} Q(x, a)^{\alpha}\right]^{\beta}+\frac{a}{\alpha} \sum_{\zeta=1}^{\infty} \frac{I_{y}\left(\frac{\zeta+1}{\alpha}, \beta+1\right)}{A^{\zeta+1} \zeta !}\right\} .
\end{array}
$$



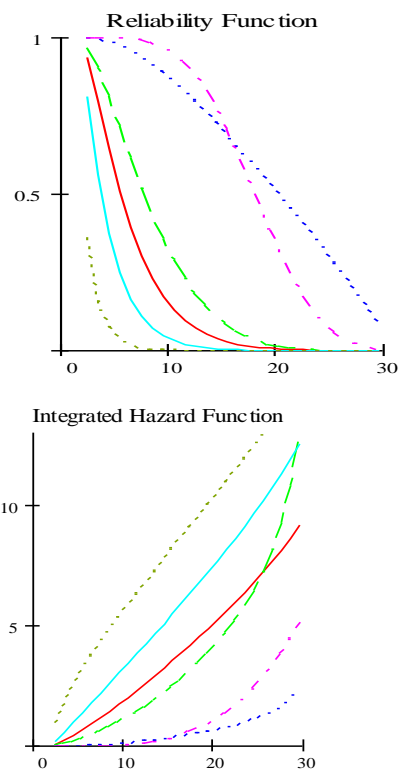

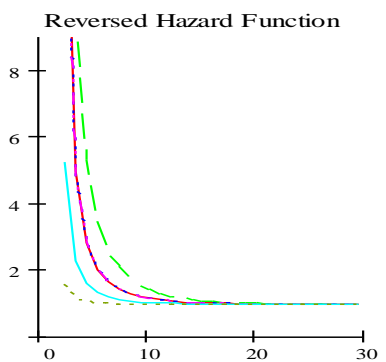

Mills Ratio

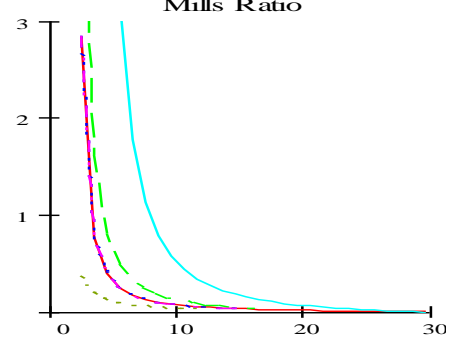

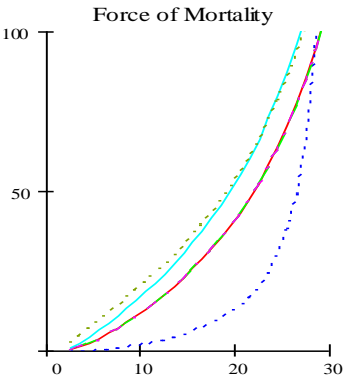

Bonferroni and Lorenz Curves

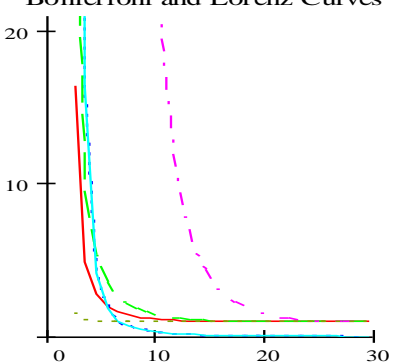

Figure 2: Reliability Measurements and Bonferroni and Lorenz Curves of $\operatorname{KRF}(a, b, \alpha, \beta)$

Figure. 2 illustrate plot the RF, FM, RHF, IHF, MR and the BLC of the $\operatorname{KRF}(a, b, \alpha, \beta)$ under different values of the shape parameters $(\alpha, \beta)$ and the scale parameters $(a, b)$. The graphs show that, the integrated hazard function and the force of mortality are increasing functions, while the reliability function and mills ratio are decreasing as the time increasing. On the other hand, the reversed hazard function and the Bonferroni and Lorenz curves are beginning decreasing functions until reached the point $(x=1)$ where became constant functions.

\subsection{Moment Generating Function}

Theorem 6: The moment generating function of a r.v X distributed as $\operatorname{KRF}(a, b, \alpha, \beta)$ is

$$
m_{x}(t)=\sum_{r=1}^{\infty} \frac{t^{r}}{r !}\left\{a^{r}+\frac{a^{r}}{\alpha} \sum_{\zeta=1}^{\infty} \frac{r^{\zeta+1}}{A^{\zeta+1} \zeta !} \beta\left(\frac{\zeta+1}{\alpha}, \beta+1\right)\right\} .
$$

Proof: The moment generating function of a r.v X is defined as $m_{x}(t)=\int_{a}^{b} e^{t x} f(x) d x$, then

$$
\begin{aligned}
& \int_{a}^{b} e^{t x} f(x) d x=\beta \int_{a}^{b} \frac{\alpha e^{t x}}{x} A^{\alpha} Q(x, a)^{\alpha-1}\left[1-A^{\alpha} Q(x, a)^{\alpha}\right]^{\beta-1} d x \\
& =\alpha \beta \sum_{r=1}^{\infty} \frac{t^{r} b}{r !} \int_{a}^{r-1} A^{\alpha} Q(x, a)^{\alpha-1}\left[1-A^{\alpha} Q(x, a)^{\alpha}\right]^{\beta-1} d x \\
& =\sum_{r=1}^{\infty} \frac{t^{r}}{r !}\left\{a^{r}+\frac{a^{r}}{\alpha} \sum_{\zeta=1}^{\infty} \frac{r^{\zeta+1}}{A^{\zeta+1} \zeta !} \beta\left(\frac{\zeta+1}{\alpha}, \beta+1\right)\right\}
\end{aligned}
$$

Therefore

$$
m_{x}(t)=\sum_{r=1}^{\infty} \frac{t^{r}}{r !}\left\{a^{r}+\frac{a^{r}}{\alpha} \sum_{\zeta=1}^{\infty} \frac{r^{\zeta+1}}{A^{\zeta+1} \zeta !} \beta\left(\frac{\zeta+1}{\alpha}, \beta+1\right)\right\}
$$

, and the Characteristic function of a

r.v X distributed as $K R F(a, b, \alpha, \beta)$ is

$$
\varphi_{x}(t)=\sum_{r=1}^{\infty} \frac{(i t)^{r}}{r !}\left\{a^{r}+\frac{a^{r}}{\alpha} \sum_{\zeta=1}^{\infty} \frac{r^{\zeta+1}}{A^{\zeta+1} \zeta !} \beta\left(\frac{\zeta+1}{\alpha}, \beta+1\right)\right\}=m_{x}(i t)
$$




\subsection{Laplace-Stieltjes Transform}

Theorem 7: The Laplace-Stieltjes transform of a r.v X distributed as $\operatorname{RRF}(a, b, \alpha, \beta)$ is given by

$$
f^{*}(s)=\sum_{r=1}^{\infty} \frac{(-s)^{r}}{r !}\left\{a^{r}+\frac{a^{r}}{\alpha} \sum_{\zeta=1}^{\infty} \frac{r^{\zeta+1}}{A^{\zeta+1} \zeta !} \beta\left(\frac{\zeta+1}{\alpha}, \beta+1\right)\right\} .
$$

Proof: Laplace-Stieltjes transform is defined by

$$
\begin{aligned}
& f^{*}(s)=\int_{a}^{b} e^{-s x} f(x) d x \text {. Then } \\
& \left.\left.\int_{a}^{b} e^{-s x} f(x) d x=\beta \int_{a}^{b} \frac{\alpha e^{-s x}}{x} A^{\alpha} Q(x, a)^{\alpha-1}\left[1-A^{\alpha} Q(x, a)^{\alpha}\right]^{\beta-1} d x=\alpha \beta \sum_{r=1}^{\infty} \frac{(-s)^{r} b \int_{a}^{b} x^{r-1} A^{\alpha} Q(x, a)^{\alpha-1}\left[1-A^{\alpha} Q(x, a)^{\alpha}\right]^{\beta-1} d x}{r !} d x\right)\right\} \\
& =\sum_{r=1}^{\infty} \frac{(-s)^{r}}{r !}\left\{a^{r}+\frac{a^{r}}{\alpha} \sum_{\zeta=1}^{\infty} \frac{r^{\zeta+1}}{A^{\zeta+1} \zeta !} \beta\left(\frac{\zeta+1}{\alpha}, \beta+1\right)\right\} \sum_{r=1}^{\infty} \frac{(-s)^{r}}{r !}\left\{a^{r}+\frac{a^{r}}{\alpha} \sum_{\zeta=1}^{\infty} \frac{r^{\zeta+1}}{A^{\zeta+1} \zeta !} \beta\left(\frac{\zeta+1}{\alpha}, \beta+1\right)\right\}=m_{x}(-s) \\
& \text { Therefore }
\end{aligned}
$$

\subsection{Estimation of Parameters}

Let $X_{1}, X_{2}, \ldots, X_{n}$ be a random sample of size $\mathrm{n}$, distributed as $K R F(a, b, \alpha, \beta)$ with likelihood function

$$
L=\left(\alpha \beta A^{\alpha}\right)^{n} \prod_{i-1}^{n}\left\{x_{i}^{-1} Q\left(x_{i}, a\right)^{\alpha-1}\left[1-A^{\alpha} Q\left(x_{i}, a\right)^{\alpha}\right]^{\beta}\right\}
$$

Then the log likelihood function $(l)$ is given by

$$
l=n \ln (\alpha \beta)+n \alpha \ln A+\sum_{i=1}^{n}\left\{(\alpha-1) \ln Q\left(x_{i}, a\right)+(\beta-1) \ln \left[1-\varsigma\left(x_{i}, a, b\right)^{\alpha}\right]-\ln x_{i}\right\} \rightarrow(8)
$$

where, $\varsigma\left(x_{i}, a, b\right)=A Q\left(x_{i}, a\right)$. By taking the partial derivatives of Eq.8 with respect to the four unknown parameters $(a, b, \alpha, \beta)$, the four normal equations are obtained as

$$
\begin{gathered}
\frac{\partial l}{\partial a}=\frac{1}{a}\left\{n \alpha A-\sum_{i=1}^{n}\left\{(\alpha-1) Q\left(x_{i}, a\right)^{-1}+\alpha(\beta-1) A\left(1-\varsigma\left(x_{i}, a, b\right)^{-1}\right)\left(1-\varsigma\left(x_{i}, a, b\right)^{-\alpha}\right)^{-I}\right\}\right\} \\
\frac{\partial l}{\partial b}=\frac{-1}{b}\left\{n \alpha A+\alpha(\beta-1) \sum_{i=1}^{n} A\left(1-\varsigma\left(x_{i}, a, b\right)^{-\alpha}\right)^{-I}\right\} \\
\frac{\partial l}{\partial \alpha}=\left\{\frac{n}{\alpha}+n \ln A+\sum_{i=1}^{n}\left\{\ln Q\left(x_{i}, a\right)+(\beta-1)\left(1-\varsigma\left(x_{i}, a, b\right)^{-\alpha}\right)^{-I} \ln \varsigma\left(x_{i}, a, b\right)\right\}\right\} \\
\frac{\partial l}{\partial \beta}=\left\{\frac{n}{\beta}+\sum_{i=1}^{n} \ln \left(1-\varsigma\left(x_{i}, a, b\right)^{-\alpha}\right)\right\}
\end{gathered}
$$

The solution of the above normal equations cannot be obtained in closed form, so MathCad 15 package can be used to get the MLE of the unknown parameters. Therefore, the elements of the Fisher information matrix $(F I)$ for the MLE can be obtained as the expectations of the negative of the second partial derivatives, and the asymptotic variance-covariance matrix $V(\underline{\eta})$ for the MLE is defined as the inverse of the Fisher's information matrix i.e., 
Where,

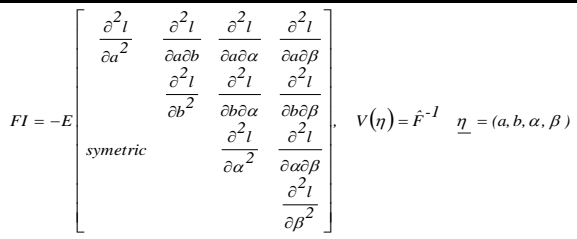

$$
\begin{aligned}
& \frac{\partial^{2} l}{\partial a^{2}}=\frac{1}{a^{2}}\left\{n \alpha A(A-1)-\sum_{i=1}^{n}\left\{(\alpha-1) Q\left(x_{i}, a\right)^{-2}\left(1-Q\left(x_{i}, a\right)\right)+\alpha A(\beta-1)\left(1-\varsigma\left(x_{i}, a, b\right)^{-1}\right)\left(1-\varsigma\left(x_{i}, a, b\right)^{-\alpha}\right)^{-2}\right\}\right. \\
& \left.\left[\left(1-\varsigma\left(x_{i}, a, b\right)^{-\alpha}\right)\left[A\left(\varsigma\left(x_{i}, a, b\right)^{-1}+1\right)-1\right]-\alpha A \varsigma\left(x_{i}, a, b\right)^{-\alpha}\left(1-\varsigma\left(x_{i}, a, b\right)^{-1}\right)\right]\right\} \\
& \frac{\partial^{2} l}{\partial b^{2}}=\frac{1}{b^{2}}\left\{n \alpha A(A+1)+\sum_{i=1}^{n}\left\{\alpha A(\beta-1)\left(1-\varsigma\left(x_{i}, a, b\right)^{-\alpha}\right)^{-2}\right\} \cdot\left[\left(1-\varsigma\left(x_{i}, a, b\right)^{-\alpha}\right)[A+1]-\alpha A \varsigma\left(x_{i}, a, b\right)^{-\alpha}\right]\right\} \\
& \frac{\partial^{2} l}{\partial \alpha^{2}}=\left\{\frac{-n}{\alpha^{2}}+(\beta-1) \sum_{i=1}^{n}\left\{\varsigma\left(x_{i}, a, b\right)^{-\alpha}\left(1-\varsigma\left(x_{i}, a, b\right)^{-\alpha}\right)^{-2}\left(\ln \varsigma\left(x_{i}, a, b\right)\right)^{2}\right\}\right\} \\
& \frac{\partial^{2} l}{\partial \beta^{2}}=\frac{-n}{\beta^{2}} \\
& \frac{\partial^{2} l}{\partial a \partial b}=\frac{-1}{a b}\left\{n \alpha A^{2}+\alpha A^{2}(\beta-1) \sum_{i=1}^{n}\left(1-\varsigma\left(x_{i}, a, b\right)^{-\alpha}\right)^{-2}\left\{l-\varsigma\left(x_{i}, a, b\right)^{-1}\left[1+\alpha\left(1-\varsigma\left(x_{i}, a, b\right)^{-1}\right)\right]\right\}\right\} \\
& \frac{\partial^{2} l}{\partial a \partial \alpha}=\frac{1}{a}\left\{n A+\sum_{i=1}^{n}\left[A(\beta-1)\left(1-\varsigma\left(x_{i}, a, b\right)^{-1}\right)\left(1-\varsigma\left(x_{i}, a, b\right)^{-\alpha}\right)^{-2}\left\{1-\varsigma\left(x_{i}, a, b\right)^{-1}\left[1+\alpha \ln \varsigma\left(x_{i}, a, b\right)\right]\right\}-Q\left(x_{i}, a\right)^{-1}\right]\right\} \\
& \frac{\partial^{2} l}{\partial a \partial \beta}=\frac{\alpha A}{a} \sum_{i=1}^{n}\left(1-\varsigma\left(x_{i}, a, b\right)^{-l}\right)\left(1-\varsigma\left(x_{i}, a, b\right)^{-\alpha}\right)^{-I} \\
& \frac{\partial^{2} l}{\partial b \partial \alpha}=\frac{-A}{b}\left\{n+(\beta-1) \sum_{i=1}^{n}\left(1-\varsigma\left(x_{i}, a, b\right)^{-\alpha}\right)^{-2}\left(1-\varsigma\left(x_{i}, a, b\right)^{-\alpha}\left(1-\alpha \ln \varsigma\left(x_{i}, a, b\right)\right)\right)\right\} \\
& \frac{\partial^{2} l}{\partial b \partial \beta}=\frac{-\alpha A}{b} \sum_{i=1}^{n}\left(1-\varsigma\left(x_{i}, a, b\right)^{-\alpha}\right)^{-I} \\
& \frac{\partial^{2} l}{\partial \alpha \partial \beta}=\sum_{i=1}^{n}\left(1-\varsigma\left(x_{i}, a, b\right)^{-\alpha}\right)^{-I} \ln \varsigma\left(x_{i}, a, b\right)
\end{aligned}
$$

\section{Application}

Suppose that $Y$ is a count response variable that follows the EWGD in Equation (2.2) and $Y$ is associated with a set of predictors. We wish to fit the response variable $Y$ by using the predictors. Suppose we have a $k-1$ row vector of predictors $x_{i}=\left(x_{i 0}=1, x_{i 1}, x_{i 2}, \ldots, x_{i,(k-1)}\right)$.

In this section, four applications to real data sets are provided to illustrate the flexibility and the usefulness of the new distribution. The first data represents the rainfall of the city of Zlitan-Libya for the period from 1980 to 2009 from the records of the five meteorological center, the data set is: $(0.122,0.122,0.139,0.146,0.168,0.17,0.178,0.18,0.183,0.184,0.192$, $0.199,0.208,0.21,0.212,0.241)$. The second data of the air conditioning system of an airplane originally presented by Linhart and Zucchini (1986), the data set is: $(0.0417,0.0655$, $0.0655,0.0655,0.0714,0.0833,0.0833,0.0833,0.0952,0.0952,0.125,0.1190,0.1369$, $0.2500,0.2798,0.3095,0.3690,0.4226,0.4226,0.5179,0.5655,0.5357,0.7143,0.7143$ ) is the failure times (week) of the air conditioning system of an airplane. 
The third data of the Survival time of infected with virulent tubercle bacilli was reported by Haq 2016, the data set is: $(1.02,1.05,1.07,1.08,1.08,1.09,1.12,1.13,1.15,1.16,1.20,1.21$, $1.22,1.22,1.24,1.3,1.34,1.36,1.39,1.44,1.46,1.53,1.59,1.60,1.63,1.63,1.68,1.71,1.72$, $1.76,1.83,1.95,1.96,1.97,2.02,2.13,2.15,2.16,2.22,2.30,2.31,2.40,2.45,2.51,2.53,2.54$, $2.54,2.78,2.93,3.27,3.42,3.47,3.61,4.02,4.32,4.58,5.55)$. The fourth data set was provided by Ed Fuller of the NICT Ceramics Division in December 1993. It contains polished window strength data (proprotions), the maximum value is 45.38 , the data set is: $(0.41,0.46,0.48$, $0.51,0.51,0.53,0.54,0.56,0.56,0.570 .59,0.59,0.59,0.6,0.61,0.66,0.69,0.73,0.74,0.74$, $0.75,0.77,0.79,0.79,0.81,0.82,0.82,0.87,0.97,1.00)$.

Some measures of goodness of fit are used, including Kolomgrof-Smirnof test $\left(\boldsymbol{K} \_S\right)$, Akaike information criterion $(\boldsymbol{A I C})$, consistent Akaike information criterion $(\boldsymbol{C A I C})$, Bayesian information criterion $(\boldsymbol{B I C})$, Anderson Darling $(\boldsymbol{A D})$ and Cramrvon Mises $(\boldsymbol{C V M})$ statistics for assessing that the data sets follow the ${ }^{K R F(a, b, \alpha, \beta)}$ and they are:

$$
\begin{aligned}
& A I C=-2 L+2 K, B I C=-2 L+K \log (n), C A I C=A I C+\frac{2 K(K+1)}{n-K-1}, \\
& A D=-n-\left(\frac{1}{n}\right) \sum_{i=1}^{n}\left\{(2 i-1)\left[\log \left(F_{i}(x)\right)+\log \left(F_{n-i+1}(x)\right)\right], C V M=\frac{1}{12 n}+\sum_{i=1}^{n}\left\{F_{i}(x)-\frac{2 i-1}{2 n}\right\}\right.
\end{aligned}
$$

where $\mathrm{n}$ is the sample size, $\mathrm{k}$ is the number of parameters of each statistical model, $\mathrm{F}(\mathrm{x})$ is the $\mathrm{CDF}$ of each statistical model and $\mathrm{L}$ is the maximized value of the log-likelihood function under the considered model. The values of the $K \_S, A I C, C A I C, B I C, A D$ and $C V M$ are represented in Table. 2 for the new proposed model compared with the other sub models: Reciprocal $(\boldsymbol{R D})$, Power Function $(\boldsymbol{P F D})$, exponential $(\boldsymbol{E x p D})$, Weibul (WD), Rayleigh $(\boldsymbol{R a y D})$ and Pareto $(\boldsymbol{P D})$ distribution. Moreover, ML estimates with confidence intervals for $(a, b, \alpha, \beta)$

based on the data sets are calculated. Mean Square Error (MSE) with variance of these estimates are represented in Table.3. Also, moments about origin, incomplete moments, probability weighted moments, Laplace-Stieltjes transform and mean residual life at the mission time $\boldsymbol{t}$ of the 4-kumaraswamy reciprocal family are calculated and represented in Table.4. In addition, mean, median, $1^{\text {st }}$ quartile, $3^{\text {rd }}$ quartile, bowley skewness (BS), moors kurtosis (MK) and mean residual life (MRL) of the four kumaraswamy reciprocal family are calculated and represented in Table.5.

\begin{tabular}{|c|c|c|c|c|c|c|}
\hline \multicolumn{7}{|c|}{ DATA I: $a=0.01(0.015), b=0.08(0.326), \alpha=0.41(1.343), B=0.90(0.384)$} \\
\hline Model & $A D$ & CVM & CAIC & $B I C$ & $A I C$ & $K \_S$ \\
\hline KRD & $7.088-$ & 0.938 & $5.110 \mathrm{E}+9-$ & $5.110 \mathrm{E}+9-$ & $5.110 \mathrm{E}+9-$ & 0.389 \\
\hline$R D$ & $4.498-$ & 2.256 & 4.1810E+4- & 4.1810E+4- & 4.1810E+4- & 0.616 \\
\hline$P F D$ & $6.898^{-}$ & 1.029 & $10.503^{-}$ & $11.585^{-}$ & -10.789 & 0.421 \\
\hline ExpD & 2.698 & 4.242 & 2.286 & 1.204 & 2.000 & 0.912 \\
\hline$W D$ & 4.570 & 4.499 & 4.923 & 2.408 & 4.000 & 0.933 \\
\hline $\operatorname{RayD}$ & 11.006 & 4.995 & 2.286 & 1.204 & 2.000 & 0.968 \\
\hline$P D$ & $4.827-$ & 2.128 & 4.923 & 2.408 & 4.000 & 0.645 \\
\hline \multicolumn{7}{|c|}{ DATA II: $a=0.01(0.183), b=0.224(1.07), \alpha=1.35(1.922), B=0.7(0.774)$} \\
\hline Model & $A D$ & $C V M$ & CAIC & $B I C$ & AIC & $K \_S$ \\
\hline KRD & $15.641-$ & 0.523 & 1.088 & 2.603 & 0.512 & 0.115 \\
\hline$R D$ & $14.655^{-}$ & 35.306 & 12.935 & 11.444 & 12.49 & 2.156 \\
\hline$P F D$ & $12.346^{-}$ & 2.419 & 11.74 & 11.074 & 11.597 & 0.482 \\
\hline ExpD & $13.242^{-}$ & 1.786 & 48.578 & 47.913 & 48.436 & 0.461 \\
\hline$W D$ & $9.237^{-}$ & 3.905 & 62.906 & 61.416 & 62.462 & 0.669 \\
\hline RayD & $2.874^{-}$ & 6.141 & 113.806 & 113.141 & 113.664 & 0.763 \\
\hline$P D$ & $3.631^{-}$ & 6.018 & 113.46 & 111.97 & 113.015 & 0.77 \\
\hline \multicolumn{7}{|c|}{ DATA III: $a=0.011(0.025), b=0.08(0.164), \alpha=0.91(1.323), B=0.091(0.526)$} \\
\hline Model & $A D$ & $C V M$ & CAIC & $B I C$ & $A I C$ & $K \_S$ \\
\hline KRD & $29.1149-$ & 1.2508 & 7.1375 & 5.3917 & 6.3682 & 0.1489 \\
\hline$R D$ & $26.0807-$ & 2.963 & 50.546 & 49.8355 & 50.3237 & 0.4631 \\
\hline$P F D$ & -16.8794 & 6.9113 & 31.8239 & 31.5071 & 31.7512 & 0.5678 \\
\hline $\operatorname{ExpD}$ & $28.4839^{-}$ & 1.5806 & 11.6515 & 11.3346 & 11.5787 & 0.2751 \\
\hline$W D$ & $19.3828^{-}$ & 6.4188 & 62.9427 & 62.2322 & 62.7205 & 0.544 \\
\hline
\end{tabular}

Table 2: The ML Estimates, $A D, C V M, C A I C, B I C, A I C, K \_S$ for the four Data Sets 
112 Four Parameters Kumaraswamy Reciprocal Family Of Distributions

\begin{tabular}{|c|c|c|c|c|c|c|}
\hline RayD & 28.2062 & 16.5944 & 323.0255 & 322.7087 & 322.9528 & 0.8638 \\
\hline$P D$ & 15.0782 & 15.5837 & 191.7351 & 191.0247 & 191.5129 & 0.877 \\
\hline \multicolumn{7}{|c|}{ DATA IV: $a=0.1(0.183), b=0.224(1.07), \alpha=1.35(1.922), B=0.7(0.774)$} \\
\hline Model & $A D$ & $C V M$ & CAIC & $B I C$ & AIC & $K \_S$ \\
\hline$K R D$ & $15.641^{-}$ & 0.523 & 1.088 & $2.603^{-}$ & $0.512^{-}$ & 0.213 \\
\hline$R D$ & $15.001^{-}$ & 35.306 & 4.416 & 2.926 & 3.971 & 2.156 \\
\hline$P F D$ & $12.346^{-}$ & 2.419 & 2.126 & 1.461 & 1.984 & 0.482 \\
\hline ExpD & $13.242^{-}$ & 1.786 & 2.143 & 1.477 & 2.000 & 0.461 \\
\hline$W D$ & $9.237^{-}$ & 3.905 & 4.444 & 2.954 & 4.000 & 0.669 \\
\hline RayD & $2.874^{-}$ & 6.141 & 2.143 & 1.477 & 2.000 & 0.763 \\
\hline$P D$ & $3.631^{-}$ & 6.018 & 4.444 & 2.954 & 4.000 & 0.770 \\
\hline
\end{tabular}

The results in Table. 2 indicates that, the values of the $K-S$ is very small for the $\operatorname{KRF}(a, b, \alpha, \beta)$ distribution, and according to the other criteria $A I C, C A I C, B I C, A D$ and $C V M$, it is noted that the ${ }_{\operatorname{KRF}(a, b, \alpha, \beta)}$ yields a better fit to all the four data sets than the other distributions. So, the ${ }^{\operatorname{KRF}(a, b, \alpha, \beta)}$ could be chosen as the best model for the four data sets.

Table 3: MLE, MSE and C.I of the unknown parameters of the $\operatorname{KRF}(a, b, \alpha, \beta)$

\begin{tabular}{|c|c|c|c|c|c|c|}
\hline \multicolumn{7}{|c|}{ DATA I: Rainfall of the city of Zlitan } \\
\hline Para. & $\begin{array}{l}\text { Init. } \\
\text { values }\end{array}$ & $M L E$ & $M S E$ & Variance & $L B C I$ & $U B C I$ \\
\hline$a$ & 0.015 & 0.01818 & $1.014 \mathrm{E}-5$ & $1.255 \mathrm{E}-5$ & 0.01816 & 0.01821 \\
\hline$b$ & 0.1 & 0.100004 & $2.14 \mathrm{E}-12$ & $2.535 \mathrm{E}-5$ & 0.09995 & 0.10005 \\
\hline$\alpha$ & 4.75 & 4.65942 & $8.205 \mathrm{E}-3$ & 0.54564 & 3.58996 & 5.72888 \\
\hline$\beta$ & 1.002 & 1.00444 & $5.976 \mathrm{E}-6$ & 0.01283 & 0.97931 & 1.02958 \\
\hline \multicolumn{7}{|c|}{ DATA II: Air conditioning system of an airplane } \\
\hline Para. & ${ }_{\text {values }}$ Init. & $M L E$ & $M S E$ & Variance & $L B C I$ & $U B C I$ \\
\hline$a$ & 0.0101 & 0.0102 & $110 \mathrm{E}-8$ & $2.7517 \mathrm{E}-$ & 0.01015 & 0.01025 \\
\hline$b$ & 0.2 & 0.02001 & 0.0324 & 0.02191 & 0.02290 & 0.06296 \\
\hline$\alpha$ & 0.625 & 0.6334 & $7.05 \mathrm{E}-5$ & 0.01161 & 0.61064 & 0.65616 \\
\hline$\beta$ & 0.4 & 0.4159 & $2.52 \mathrm{E}-4$ & $5.845 \mathrm{E}-3$ & 0.40444 & 0.42736 \\
\hline \multicolumn{7}{|c|}{ DATA III: Survival time of infected with virulent tubercle bacilli } \\
\hline Para. & $\begin{array}{l}\text { Init. } \\
\text { values }\end{array}$ & $M L E$ & $M S E$ & Variance & $L B C I$ & $U B C I$ \\
\hline$a$ & 0.211 & 0.21448 & $1.209 \mathrm{E}-5$ & $4.925 \mathrm{E}-3$ & 0.20483 & 0.22413 \\
\hline$b$ & 0.335 & 0.33521 & $4.387 \mathrm{E}-8$ & $8.609 \mathrm{E}-3$ & 0.31833 & 0.35208 \\
\hline$\alpha$ & 0.4 & 0.39456 & $2.958 \mathrm{E}-5$ & $2.875 \mathrm{E}-3$ & 0.38893 & 0.4002 \\
\hline$\beta$ & 0.5 & 0.48743 & $1.578 \mathrm{E}-4$ & $4.529 \mathrm{E}-3$ & 0.47856 & 0.49631 \\
\hline \multicolumn{7}{|c|}{ DATA IV: Polished window strength data } \\
\hline Para. & values $^{\text {Init. }}$ & $M L E$ & MSE & Variance & $L B C I$ & $U B C I$ \\
\hline$a$ & 0.113 & 0.21155 & $9.711 \mathrm{E}-3$ & $1.383 \mathrm{E}-3$ & 0.20884 & 0.21426 \\
\hline$b$ & 0.219 & 0.46465 & 0.06034 & $1.539 \mathrm{E}-3$ & 0.46163 & 0.46767 \\
\hline$\alpha$ & 1.75 & 1.83947 & $8.005 \mathrm{E}-3$ & 0.11677 & 1.6106 & 2.06834 \\
\hline$\beta$ & 0.739 & 0.75661 & $3.099 \mathrm{E}-4$ & 0.01299 & 0.73114 & 0.78207 \\
\hline
\end{tabular}

$\operatorname{KRF}(a, b, \alpha, \beta)$

\begin{tabular}{|c|c|c|c|c|c|c|c|}
\hline \multicolumn{2}{|c|}{$\begin{array}{l}\text { DATA I: Rainfall of the city } \\
\text { of Zlitan-Libya }\end{array}$} & \multicolumn{2}{|c|}{$\begin{array}{l}\text { DATA II: Air conditioning } \\
\text { system of an airplane }\end{array}$} & \multicolumn{2}{|c|}{$\begin{array}{l}\text { DATA III: Survival time of } \\
\text { infected with virulent tubercle } \\
\text { bacilli }\end{array}$} & \multicolumn{2}{|c|}{$\begin{array}{cc}\text { DATA } \quad I V: & \text { Polished } \\
\text { window strength data } & \end{array}$} \\
\hline measue & Value & measure & Value & measue & Value & measue & Value \\
\hline Mean & 0.0761 & Mean & 0.09729 & Mean & 0.27111 & Mean & 0.37366 \\
\hline$Q(0.25)$ & 0.06442 & $Q(0.25)$ & 0.02758 & $Q(0.25)$ & 0.2272 & $Q(0.25)$ & 0.32224 \\
\hline$Q(0.5)$ & 0.07893 & $Q(0.5)$ & 0.08658 & $Q(0.5)$ & 0.26775 & $Q(0.5)$ & 0.38393 \\
\hline$Q(0.75)$ & 0.09023 & $Q(0.75)$ & 0.16948 & $Q(0.75)$ & 0.31476 & $Q(0.75)$ & 0.43273 \\
\hline Variance & $2.83 \mathrm{E}-4$ & Variance & $4.82 \mathrm{E}-3$ & Variance & $1.605 \mathrm{E}-3$ & Variance & $4.44 \mathrm{E}-3$ \\
\hline Skewnes & -0.5971 & Skewness & 0.06296 & Skewness & -2.06955 & Skewness & -0.4428 \\
\hline $\begin{array}{c}\text { Bowley } \\
\text { Skewness }\end{array}$ & -0.1243 & Bowley Skewness & 0.16838 & Bowley Skewness & 0.07386 & Bowley Skewness & -0.1167 \\
\hline kurtosis & 2.52304 & kurtosis & 1.3105 & kurtosis & 95.51749 & kurtosis & 1.83106 \\
\hline Moors Kurtosis & 1.10851 & Moors Kurtosis & 0.70378 & Moors Kurtosis & 0.74458 & Moors Kurtosis & 1.02842 \\
\hline
\end{tabular}

Based on the simulation study, can be concluded that the maximum likelihood estimators are appropriate for estimating the $\operatorname{KR} F(a, b, \alpha, \beta)$. All the real data provides a better fit to the $\operatorname{KRF}(a, b, \alpha, \beta)$ and estimate the true parameters $(a, b, \alpha, \beta)$ well with relatively small $M S E$ and reduce towards zero, with short confidence intervals. Moreover, the mean residual life 
decreases when the mission time $(t)$ increases as shown from the results in Table.3. In general, the proposed model and the asymptotic approximation work well under the situation and provides a better fit for the real data. So, the $\operatorname{KRF}(a, b, \alpha, \beta)$ very useful in many fields and has many benefits especially in practical life. Therefore, it can be applied to several realistic data and chosen as a suitable model for lifetime data.

\section{Conclusions}

This paper presented a new distribution named the four parameters kumaraswamy reciprocal family abbreviate by $\operatorname{KR} F(a, b, \alpha, \beta)$. The maximum likelihood method is applied for estimating the model parameters. Nine special models namely, reciprocal, beta, uniform, power function, exponential, negative exponential, weibull, rayleigh and pareto distribution are provided. Further, the derived properties of the $\operatorname{KRF}(a, b, \alpha, \beta)$ are valid to the selected model, such as, quantile function, median, random numbers, bowley skewness, moors kurtosis, reliability function, force of mortality, reversed hazard function, integrated hazard function, mills ratio, mean residual life, moments about origin, incomplete moments, probability weighted moments, bonferroni and lorenz curves, moment generating function, characteristic function, laplace-stieltjes transform with estimation of parameters. It also explained the behavior of the reliability function, force of mortality, mills ratio, reversed hazard function, integrated hazard function, bonferroni and lorenz curves. Four real life applications have also presented for explaining the better fit and the benefit of the observed model in many practical life. The better fit and the benefit of the new model is illustrated by four real data sets and the results of the applications nicely exhibit the fact that the $\operatorname{KRF(a,b,\alpha ,\beta )}$ provides a better fit than others sub models in many. And it performs better and a suitable in many situations and can be applied to several realistic data.

\section{References}

[1] Fuller.Jr.E.R., Frieman.S.W., Quinn. J.B., Quinn.G.D. and Carter.W.C. : Fracture mechanics approach to the design of glass aircraft windows, SPIE Proc., 2286, 419-430,1994.

[2] Hamming.R.W. : On the distribution of numbers, The Bell System Technical Journal, 49(8) 16091625,1970 .

[3] Hassan.S. and Elgarhy. M. : Kumaraswamy Weibull-generated family of distributions with applications, Advances and Applications in Statistics, 48,205-239,2016.

[4] Haq.M.A. : Kumaraswamy Exponentiated Inverse Rayleigh Distribution. Mathematical Theory and Modeling, 6, 93-104,2016.

[5] Linhart.H. and Zucchini.W. : Model selection, New York: Wiley.1986.

[6] Rama.S. and Mishra.A. : A quasi Lindley distribution .African Journal of Mathematics and Computer Science Research,6(4), 64-71,2013. 
[7] Salma Omar Bleed : L-Quadratic Distribution. International Journal of Computer Applications, 179(13),6-11,2018.

[8] Salma Omar Bleed and Arwa E.A. Abdelali : Transmuted Arcsine Distribution Properties and Application, International Journal of Research - Granthaalayah, 6(10), 38-47,2018.

[9] Shawki.A.W. and Elgarhy.M. : Kumaraswamy SUSHILA Distribution. International Journal of Scientific Engineering and Science, 1(7), 29-32,2017. 